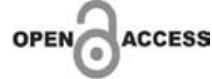

\title{
Ligadura Interesfincteriana do Trato Fistuloso (LIFT) para Fístulas Anais: Uma Experiência Brasileira Bi- Institucional
}

\author{
Intersfincterial Ligation of Fistula Tract (LIFT) for Patients with \\ Anal Fistulas: A Brazilian Bi-Institutional Experience
}
Carlos Ramon Silveira Mendes ${ }^{1 *}$, Andre Luiz Santos ${ }^{1}$, Joana Carolina Saraiva de Paula Pessoa $^{1}$, Eduardo Costa Cobas ${ }^{1}$, Sergio Eduardo Alonso Araujo ${ }^{1}$, Meyline Andrade Lima ${ }^{1}$

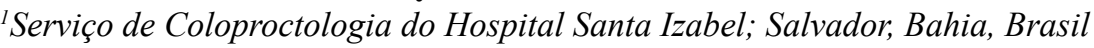

Correspondence addresses: Dr. Carlos R.S. Mendes marcos.almeida@hotmail.com

Received: October 24, 2019

Revised: October 30, 2019

Accepted: October 30, 2019

Published: December 2 ${ }^{\text {nd }}, 2019$

Data Availability Statement: All relevant data are within the paper and its Supporting Information files.

Funding: This work was the result of authors' initiative. There was no support of research or publication funds.

Competing interests: The authors have declared that no competing interests exist.

\section{Copyright}

(C) 2019 by Santa Casa de Misericórdia da Bahia.

All rights reserved.

ISSN: 2526-5563
O melhor tratamento para a fístula anal deve eliminar a infecção e promover a cicatrização do trato, preservando o esfíncter anal e a continência completa. $O$ objetivo do presente estudo foi o de determinar a taxa de sucesso após o uso da técnica modificada de ligadura interesfincteriana do trato fistuloso (LIFT) para pacientes com fístulas anais. É um estudo de coorte observacional brasileiro biinstitucional com o procedimento LIFT modificado (ligadura interesfincteriana do trato fistuloso sem excisão). Foi estabelecida base de dados clínica para as seguintes variáveis: idade, gênero, IMC, comorbidades, distância entre o orifício externo e o ânus, operação anterior para fístula, tipo de fístula, tempo cirúrgico, complicações intra e pós-operatórias, duração do seguimento e taxa de sucesso. Entre novembro de 2015 e janeiro de 2017, 38 pacientes com fístulas transesfincterianas foram operados com o procedimento LIFT modificado. Dezessete (44,7\%) eram homens. A idade média foi de 41 (18-67) anos. $O$ IMC médio foi de $26,4(22-38) \mathrm{Kg} / \mathrm{m}^{2}$. Cinco $(13,2 \%)$ tinham sido submetidos à operação anterior. A fístula era transesfincteriana em todos os casos. $O$ acompanhamento médio foi de 32 (14-56) semanas. Sucesso foi observado em 30 (79\%) pacientes. A técnica LIFT sem excisão do trato da fístula provou ser segura e eficaz para fístulas anais transesfincterianas.

Palavras-chave: Fístula Retal; Abscesso; Ânus.

The best treatment for anal fistula should extirpate infection and promote healing of the tract, whilst preserving the anal sphincter complex and full continence. The aim of this study was to analyze the success rate after a modified technique for ligation of the intersphincteric fistula tract (LIFT) for patients with anal fistulas. This study is a prospective (observational cohort study) Brazilian bi-institutional experience with a modified (ligation of the intersphincteric fistula tract without excision) LIFT technique that was undertaken. A clinical database was settled for the following variables: age, gender, BMI, comorbidities, distance between external orifice and the anus, previous fistula surgery, type of fistula, operative time, intra- and postoperative complications, duration of follow-up, and success rate. Between November 2015 and January 2017,

Resumo do Artigo: Araújo SEA, Marcante MT, Mendes CRS, Bertoncini AB, Seid VE, Horcel LA, Perez RO, Klajner S. Ligadura Interesfincteriana do Trato Fistuloso (LIFT) para Fístulas Anais: Uma Experiência Brasileira Bi-Institucional. ABCD Arq Bras Cir Dig Original Article 2017;30(4):235-238 DOI: /10.1590/0102-6720201700040002. 
38 patients with transsphincteric fistulas were operated on using the modified LIFT procedure. Seventeen (44.7\%) were men. Median age was 41 (18-67) years. Median BMI was $26.4(22-38) \mathrm{Kg} / \mathrm{m}^{2}$. Five (13.2\%) had undergone previous surgery. The fistula was transsphincteric in all cases. Median follow-up was 32 (range, 14-56) weeks. Success was observed in $30(79 \%)$ patients. The LIFT technique without excision of the fistula tract proved to be safe and effective for transsphincteric anal fistulas.

Keywords: Anal Fistula; Abscess; Anus.

\section{Introdução}

Fístula anal é um processo infeccioso persistente que se desenvolve entre o canal anal e a pele perianal. As causas principais são devido à infecção criptoglandular, Doença de Crohn, trauma, radiação ou malignidade. As fístulas complexas têm envolvimento transesfincteriano, supraesfincteriano ou extra-esfincteriano. Elas podem ser definidas como ferradura, recorrentes, e todas as fístulas anteriores quando ocorrem em mulheres, também podem apresentar múltiplos trajetos.

O melhor tratamento para a fístula anal deve eliminar a infecção e promover a cicatrização do trato, preservando o esfíncter anal e a continência completa. A ligadura do trato interesfincteriano da fístula (LIFT) foi descrita como a inovação mais recente para a operação com preservação do esfíncter e manejo de fístulas anais complexas. Relatada pela primeira vez por Rojanasakul e colaboradores $^{1}$ em 2007 como um procedimento de preservação do esfíncter, principalmente indicado na ligadura e a excisão da porção interesfincteriana do trato da fístula, fechando a abertura interna, além de eliminar o foco séptico.

\section{Métodos}

Este é um estudo prospectivo bi-institucional de coorte realizado entre novembro de 2014 e novembro de 2015. Ele foi aprovado pelo Conselho de Revisão Institucional nas duas instituições (Hospital Israelita Albert EinsteinSão Paulo-SP; Hospital Santa Izabel-Salvador, BA). Consentimento informado por escrito para o procedimento LIFT e o acordo para participar de avaliações periódicas de acompanhamento foram obtidos para todos os pacientes incluídos.
O desfecho primário foi o sucesso após a operação usando o procedimento LIFT. Ele foi definido como a cicatrização completa da ferida interesfincteriana e do orifício externo sem qualquer sinal de recorrência. Falha foi considerada como o diagnóstico clínico de recorrência de fístula em qualquer momento no seguimento pós-operatório definido por entrevista clínica, exame físico e ressonância magnética $(\mathrm{RM})$.

Os critérios de inclusão foram fístulas anais complexas (transesfincterianas ou supraesfincterianas). Os critérios de exclusão foram fístulas superficiais, pacientes com doença de Crohn, radioterapia prévia e malignidade colorretal.

Foi criado um banco de dados para coletar das seguintes variáveis: idade, gênero, índice de massa corpórea (IMC), comorbidades, distância entre o orifício externo e o ânus, operação de fístula prévia, tipo de fístula, tempo cirúrgico, complicações intra e pós-operatórias, duração do seguimento e taxa de sucesso.

\section{Técnica Cirúrgica}

Todos os pacientes foram operados usando a técnica LIFT. Não receberam preparo intestinal ou enemas. A terapia com antibióticos começou uma hora antes da operação e a internação até a alta hospitalar foi de $24 \mathrm{~h}$. Foram operados na posição de litotomia sob anestesia espinhal combinada com sedação intravenosa. O trato da fístula foi definido. Uma pequena incisão curvilínea $(2-3 \mathrm{~cm})$ foi então criada sobre a ranhura interesfincteriana no nível do trato da fístula. Foram realizadas a dissecção sem corte do plano interesfincteriano ao nível do trato da fístula sondada. Foram separados os esfíncteres interno e externo. Foram utilizadas duas suturas 
absorvíveis (3\% de ácido poliglicólico) para proteger o trato da fístula. Realizadas ligaduras com sutura dupla do trato da fístula e realizadas secções entre estas duas suturas. Revisadas hemostasias e fechadas musculaturas com sutura de ácido poliglicólico 3/0 interrompidas. As incisões da pele foram coladas com Dermabond ${ }^{\circledR}$ a critério dos cirurgiões. As excisões dos orifícios na abertura externa foram realizadas para facilitar a drenagem e evitar o fechamento precoce do trato distal.

\section{Cuidados Pós-Operatórios}

Todos os pacientes receberam alta com prescrição de anti-inflamatórios, analgésicos narcóticos e laxativos na manhã seguinte. Foram instruídos a usar banhos de assento duas vezes ao dia e sempre após evacuação intestinal. Os acompanhamentos foram programados em intervalos de 2-4 semanas até o diagnóstico clínico de cura. Aqueles com suspeita de recorrência, a ressonância magnética foi obrigatória.

\section{Resultados}

Entre novembro de 2015 e novembro de 2017, 38 pacientes com fístulas anais recémdiagnosticadas ou recorrentes foram operados com a técnica LIFT. Dezessete $(44,7 \%)$ eram homens. A idade média foi de 41 (18-67) anos. O IMC médio foi de $26,4(22-38) \mathrm{Kg} / \mathrm{m}^{2}$. Em sete $(18,4 \%)$ observou-se uma ou mais comorbidades. Quatro $(10,5 \%)$ apresentaram hipertensão e três $(7,9 \%)$ eram diabéticos. A distância mediana entre o orifício externo e o ânus foi de 5 (3-8) $\mathrm{cm}$. Dezessete $(44,7 \%)$ foram submetidos a exame pré-operatório com RM. Cinco $(13,2 \%)$ submeteram-se à operação anterior poupando o esfíncter (excluindo incisão e drenagem de abscesso) para a fístula anal. Portanto, nesta série, cinco $(13,2 \%)$ tiveram o LIFT modificado devido à recorrência. Em três $(8 \%)$ casos, o procedimento anterior foi o plug para fístula anal (Biodesign ${ }^{\circledR}$ Fistula Plug Set, Cook Medical, Bloomington, IN, EUA) e em dois $(5,2 \%)$, a fistula anal vídeoassistida (VAAFT ${ }^{\circledR}$, Karl Storz, Tuttlingen, Alemanha). Não houve complicações intra ou pós-operatórias.

\section{Avaliação do Sucesso Cirúrgico}

A duração média do seguimento foi de 32 (1456) semanas. O sucesso foi observado em 30 (79\%) pacientes. No final do presente acompanhamento, $8(21 \%)$ apresentaram recorrência após LIFT. Em relação a esses, o intervalo de tempo médio para recorrência foi de 24,8 semanas (18-42,5). Em todos os casos de falha, o orifício externo recorrente foi observado na cicatriz de incisão cirúrgica do LIFT. Portanto, todas as fístulas recorrentes tornaramse interesfincterianas e foram reoperadas com abordagem única de fistulotomia (técnica de layopen). O seguimento após a técnica de fistulotomia para as falhas foi de seis (3-7) semanas.

\section{Discussão}

Nesta experiência bi-institucional brasileira com o LIFT, foi observado que $79 \%$ dos pacientes com fístulas anais, foram curados após procedimento sem drenagem prévia e uso de fio seton. Além disso, a operação mostrou-se segura e fácil de aprender e uma opção atrativa em países em desenvolvimento, uma vez que, não foram utilizados materiais especializados ou caros no procedimento.

Pelo menos seis variações técnicas diferentes do LIFT são descritas na literatura e nenhum estudo comparativo entre as variações foi feita para verificar a verdadeira eficácia do LIFT clássico ou qualquer uma de suas variações. A técnica utilizada em todos os pacientes foi a sem excisão do trato da fístula, que representa pequena variação do estudo original.

O sucesso foi definido objetivamente como a cicatrização pós-operatória completa do orifício externo da fístula quanto do acesso cirúrgico (incisão). Por outro lado, a falha foi definida como a saída persistente de secreção através do orifício externo da fístula original ou da ferida interesfincteriana. 
O uso de seton pré-LIFT para drenagem da fístula anal não foi usado neste estudo. Três relatos de séries de casos sobre fatores preditivos associados ao sucesso após LIFT para a fístula transenfincteriana demonstraram que o seton não afetou as taxas de sucesso.

Poucos autores obtiveram sucesso quando relataram fatores de risco para falha após o LIFT ou suas variações, impedindo assim a iniciativa de adaptar o procedimento cirúrgico ao perfil de risco de um determinado paciente. Infelizmente, a série atual foi pequena para permitir análise adequada dos fatores de risco associados à falha. No presente estudo, todas as falhas puderam ser gerenciadas com sucesso por meio de operação simples de fistulotomia.

As principais limitações deste estudo provêm do seudesignnãocomparativo, deumacompanhamento limitado e da ausência de avaliação em relação às variáveis associadas à falha após LIFT. No entanto, novas evidências ainda são necessárias para determinar o resultado em longo prazo desta técnica, seu impacto na continência e como ela se compara com outras operações para preservação do esfíncter. Estudos adicionais são necessários para identificar fatores de risco para falhas de tratamento e sua eficácia em comparação com outra operação com preservação do esfíncter para fístulas anais.

\section{Conclusão}

A técnica LIFT sem excisão do trato da fístula provou ser segura e eficaz para o tratamento de fistulas anais transesfincterianas.

\section{Referências}

1. Rojanasakul A, Pattanaarun J, Sahakitrungruang C et al. Total anal sphinctersaving technique for fistula-inano; the ligation of intersphincteric fistula tract. J Med Assoc Thail. 2007;90:581-6.

1. Abcarian AM, Estrada JJ, Park $J$ et al. Ligation of intersphincteric fistula tract: early results of a pilot study. Dis Colon Rectum. 2012;55(7):778-82.

2. Aboulian A, Kaji AH, Kumar RR. Early result of ligation of the intersphincteric fistula tract for fistulain-ano. Dis Colon Rectum 2011;54:289-92.
3. Alasari S, Kim NK. Overview of anal fistula and systematic review of ligation of the intersphincteric fistula tract (LIFT). Tech Coloproctol. 2014;18:1322.

4. Amato A, Bottini C, De Nardi P et al. Evaluation and management of perianal abscess and anal fistula: a consensus statement developed by the Italian Society of Colorectal Surgery (SICCR). Tech Coloproctol 2016;19:595-606.

5. Bleier JIS, Moloo H, Goldberg SM. Ligation of the intersphincteric fistula tract: an effective new technique for complex fistulas. Dis Colon Rectum. 2010;53:43-6.

6. Campbell ML, Abboud EC, Dolberg ME et al. Treatment of refractory perianal fistulas with ligation of the intersphincteric fistula tract: preliminary results. Am Surg. 2013;79:723-7.

7. Christoforidis D, Etzioni DA, Goldberg SM et al.Treatment of complex anal fistulas with the collagen fistula plug. Dis Colon Rectum. 2008;51:1482-7.

8. Ellis CN, Rostas JW, Greiner FG. Long-term out comes with the use of bioprosthetic plugs for the management of complex anal fistulas. Dis Colon Rectum. 2010;53:798-802.

9. Hong KD, Kang S, Kalaskar S et al. Ligation of intersphincteric fistula tract (LIFT) to treat anal fistula: systematic review and meta-analysis. Tech Coloproctol 2014;18:685-91.

10. Lehmann J-P, Graf W. Efficacy of LIFT for recurrent anal fistula. Colorectal Dis. 2013;15(5):592-5.

11. Limura E, Giordano P. Modern management of anal fistula. World J Gastroenterol. 2015;21:12-20.

12. Liu WY, Aboulian A, Kaji AH et al. Long-term results of ligation of intersphincteric fistula tract (LIFT) for fistula-in-ano. Dis Colon Rectum. 2013;56:343-7.

13. Madbouly KM, El Shazly W, Abbas KS et al. Ligation of intersphincteric fistula tract versus mucosal advancement flap in patients with high transsphincteric fistula-in-ano: a prospective randomized trial. Dis Colon Rectum. 2014;57:1202-8.

14. Meinero P, Mori L. Video-assisted anal fistula treatment (VAAFT): a novel sphincter-saving procedure for treatingcomplex anal fistulas. Tech Coloproctol. 2011;15:417-22.

15. Mendes CRS, Ferreira LS de M, Sapucaia RA et al. Video-assisted anal fistula treatment: technical considerations and preliminary results of the first Brazilian experience. ABCD Braz Arch Dig Surg. 2014;27:77-81.

16. Mushaya C, Bartlett L, Schulze B et al. Ligation of intersphincteric fistula tractcompared with advancement flap for complex anorectal fistulas requiring initial set on drainage. Am J Surg, 2012;204:283-9. 
17. Quah HM, Tang CL, Eu KW et al. Meta-analysis of randomized clinical trials comparing drainage alone $v s$ primary sphincter-cutting procedures for anorectal abscess-fistula. Int J Colorectal Dis. 2006;21:602-9.

19. Sirany A-ME, Nygaard RM, Morken JJ. The ligation of the intersphincteric fistula tract procedure for anal fistula: a mixed bag of results. Dis Colon Rectum. 2016;58:604-12.

20. Sirikurnpiboon S, Awapittaya B, Jivapaisarnpong P. Ligation of intersphincteric fistula tract and its modification: Results from treatment of complex fistula. World J Gastrointest Surg. 2013;5:123-8.

21. Steele SR, Kumar R, Feingold DL et al. Standards Practice Task Force of the American Society of Colonand Rectal Surgeons. Practice parameters for the management of perianal abscess and fistula-in-ano. Dis Colon Rectum. 2011;54:1465-74.
22. Tan K-K, Tan IJ, Lim FS et al. The anatomy of failures follow ingtheligation of intersphincteric tract technique for anal fistula: a reviewof 93 patients over 4 years. Dis Colon Rectum. 2011;54:1368-72.

23. Yassin NA, Hammond TM, Lunniss PJ et al. Ligation of the intersphincteric fistula tract in the management of anal fistula. A systematic review. Colorectal Dis. 2013;15:527-35.

24. Wallin UG, Mellgren AF, Madoff RD et al. Does ligation of the intersphincteric fistula tract raise the bar in fistula surgery? Dis Colon Rectum. 2012;55:1173-8.

25. Westerterp M, Volkers NA, Poolman RW et al. Anal fistulotomy between Skylla and Charybdis. Colorectal Dis. 2003;5:549-51.

26. Zirak-Schmidt S, Perdawood SK. Management of anal fistula by ligation of the intersphincteric fistula tract - a systematic review. Dan Med J. 2014;61:A4977. 